International Journal of Pure and Applied Mathematics

Volume 94 No. 1 2014, 9-20

ISSN: 1311-8080 (printed version); ISSN: 1314-3395 (on-line version)

url: http://www.ijpam.eu

doi: http://dx.doi.org/10.12732/ijpam.v94i1.2

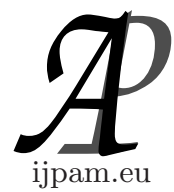

\title{
ON DERIVATION OF RATIONAL SOLUTIONS OF BABBAGE'S FUNCTIONAL EQUATION
}

\author{
Ampon Dhamacharoen ${ }^{1}$, Porntip Kasempin ${ }^{2}$ \\ ${ }^{1,2}$ Department of Mathematics \\ Burapha University \\ Bangsaen, Chonburi, 20131, THAILAND
}

\begin{abstract}
This paper investigates the functional $n^{\text {th }}$ root of the identity function in the rational form $f(x)=\frac{a x+b}{c x+d}$. The formula is analyzed via linear transformation in order to obtain the conditions for a, b, c and d. Some examples in linear rational form or in other forms are provided. The rational form of quadratic functions is also discussed.
\end{abstract}

AMS Subject Classification: 39B72, 39B12, 26C15, 39B72, 40C05 Key Words: functional root, Babbage's equation, rational form, functional equation, matrix representation

\section{Introduction}

A functional root is a function which when applied a given number of times, equals a given function. Given $f(x)$, the $n^{t h}$ ordered composite function, $f^{[n]}(x)$ can be computed by applying the function $f, n$ times. The formal definition can be written as:

$$
f^{[n]}(x)=f\left(f^{[n-1]}(x)\right), \quad n=1,2,3, \ldots,
$$

where $f^{[0]}(x)=x$, [6]. If the function $g(x)$ is given, the functional $n^{\text {th }}$ root of

Received: August 19, 2013

(C) 2014 Academic Publications, Ltd.

$\S$ Correspondence author url: www.acadpubl.eu 
$g$ is the solution of the functional equation

$$
f^{[n]}(x)=g(x)
$$

For example, $x^{2}+2$ is the functional $2^{\text {nd }}$ root of $x^{4}+4 x^{2}+6$. If $g(x)=x$, the equation (2) is called Babbage's equation (named after Babbage, 1791-1871 $[7,8,10])$. In this paper, we concentrate on the solutions of Babbage's equation, which are the functional root of the identity function. (This root is also called the functional $n^{\text {th }}$ root of unity, or the functional $n^{\text {th }}$ root of $x$.)

The functional equation (2), and other more general functional equations, have been studied in respect to the properties of the solutions, and some specific solutions are provided. (see $[2,3,4,5,6,9,10,11]$ ). For example, the function $f(x)=x$ is the only real continuous solution of the Babbage equation when $n$ is odd. The function $f(x)=c-x$, where $c$ is a constant, $f(x)=\sqrt{r^{2}-x^{2}}$, $0 \leq x \leq r$, and $f(x)=\frac{2 x+3}{x-2}$ are the functional second roots. These roots are also the functional $n^{\text {th }}$ root of $x$ when $n$ is even. Some of the functional third roots of unity are:

(a) $f(x)=x^{r}$, where $r=\frac{-1+\sqrt{3} i}{2}$;

(b) $f(x)=\frac{-1+\sqrt{3} i}{2} x$;

(c) $f(x)= \begin{cases}x-2 & \text {, if } 3 k-1 \leq x<3 k \\ x+1 & , \text { otherwise, }\end{cases}$ for $k=0, \pm 1, \pm 2, \pm 3, \ldots$;

(d) $f(x)=\frac{x-3}{x+1}$, where $x \neq-1$.

(a) and (b) are the complex valued functions; (c) has infinite discontinuous points; (d) is interesting since it is a real valued function, which is continuous but a single point.

In accordance with the examples above, the functional $n^{\text {th }}$ root of $x$ can be obtained using the same pattern.

(a) $f(x)=x^{r}$, where $r$ is the $n^{\text {th }}$ root of unity. 
(b) $f(x)=r x$, where $r$ is the $n^{\text {th }}$ root of unity.

(c) $f(x)= \begin{cases}x-n+1 & \text {, if } n k-1 \leq x<n k \\ x+1 & \text {, otherwise }\end{cases}$

for $k=0, \pm 1, \pm 2, \pm 3, \ldots$;

(d) $f(x)=\frac{\beta+\alpha x}{\gamma+\delta x}$, where $x \neq-\frac{\gamma}{\delta}$.

It is not difficult to verify that all functions are solutions. (d) is the rational form function where $\gamma$ and $\delta$ do not vanish at the same time. This function is a well known solution for Babbage's equation, where the condition for the unknown constant is:

$$
\delta=-\frac{\beta^{2}-2 \beta \gamma \cos \frac{2 k \pi}{n}+\gamma^{2}}{2 \alpha\left(1+\cos \frac{2 k \pi}{n}\right)}
$$

for some constant integer $k$ relatively primed to $n$ (see $[1,9]$ ).

If $f(x)$ is a functional $n^{\text {th }}$ root of the identity function, then the function $F$, defined by

$$
F(x)=g^{-1} f g(x)
$$

is also a functional root, for any function $g$ and its inverse $g^{-1}$ (see [9]). This fact is easily seen by performing the composition $\mathrm{n}$ times. Therefore, one can construct an infinite number of such functional roots. The function $F(x)$ $=\left(\frac{x^{1 / 3}-3}{x^{1 / 3}+1}\right)^{3}$ is the functional third root of this type.

Now we are interested in the derivation of the rational form solutions as in (d), and wish to investigate this formula to determine whether there is another solution of this form.

From (d), if $\delta=0$, the rational form becomes a linear function which is reduced to $x$, or $c-x$ if $n=2$. Assuming that $\delta \neq 0$, the rational form can be reduced to $\frac{a x+b}{x+d}$. Therefore, the problem is to find $a, b$ and $d$ so that $f(x)$ $=\frac{a x+b}{x+d}$ is the functional $n^{t h}$ root of $x$.

It is obvious that if $m$ and $n$ are positive integers and $m \mid n$, and if $f$ is an $m^{\text {th }}$ functional root of $x$, then $f$ is also an $n^{\text {th }}$ functional root of $x$. Here we concentrate on the $n^{\text {th }}$ root, in which $n$ is minimal; that is there is no $m<n$, such that $f$ is the $m^{\text {th }}$ root. 


\section{Matrix Representation and Derivation}

Since $x=\frac{x}{1}$ for all real numbers $x$, so it can be represented in the vector form $\left[\begin{array}{l}x \\ 1\end{array}\right]$. Thus, the rational form $f(x)=\frac{a x+b}{x+d}$ can be written in the vector form

$$
\left[\begin{array}{c}
a x+b \\
x+d
\end{array}\right]=\left[\begin{array}{ll}
a & b \\
1 & d
\end{array}\right]\left[\begin{array}{l}
x \\
1
\end{array}\right]
$$

where $\left[\begin{array}{cc}a & b \\ 1 & d\end{array}\right]$ is a $2 \times 2$ matrix.

The composite function $f(f(x))$ is $\frac{a(a x+b)+b(x+d)}{a x+b+d(x+d)}$, which can be represented in the following form:

$$
\left[\begin{array}{c}
a(a x+b)+b(x+d) \\
a x+b+d(x+d)
\end{array}\right]=\left[\begin{array}{ll}
a & b \\
1 & d
\end{array}\right]\left[\begin{array}{c}
a x+b \\
x+d
\end{array}\right]=\left[\begin{array}{ll}
a & b \\
1 & d
\end{array}\right]^{2}\left[\begin{array}{l}
x \\
1
\end{array}\right]
$$

By induction, the $n^{\text {th }}$ composite function $f^{[n]}(x)$ can be represented as $\left[\begin{array}{ll}a & b \\ 1 & d\end{array}\right]^{n}\left[\begin{array}{l}x \\ 1\end{array}\right]$

For convenience, in all further analysis, we shall give a formal definition for vector representations of rational forms.

Definition 1. The vector $\left[\begin{array}{l}u \\ v\end{array}\right]$ is a representation of a number $x$ if $x=$ $\frac{u}{v}$, where $u$ and $v$ are real numbers and $v \neq 0$.

Definition 2. We say that the two vectors $\mathbf{x}$ and $\mathbf{y}$ are rational equivalent, written $\mathbf{x} \sim \mathbf{y}$, if both $\mathbf{x}$ and $\mathbf{y}$ are representations of the same number.

The followings are useful properties which are easily obtained from the definitions:

Property 1. Let $\mathbf{x}$ and $\mathbf{y}$ be vectors in $R^{2}$, then $\mathbf{x} \sim \mathbf{y}$ if and only if $\mathbf{x}=$ $k \mathbf{y}$ for some numbers $k \neq 0$.

Property 2. A rational equivalent is an equivalent relation.

Property 3. $\left[\begin{array}{l}x \\ 1\end{array}\right]$ is a representation of $x$. If $v \neq 0$, then $\left[\begin{array}{l}u \\ v\end{array}\right] \sim\left[\begin{array}{l}x \\ 1\end{array}\right]$ if and only if $x=\frac{u}{v}$. 
Property 4. Each vector $\left[\begin{array}{l}u \\ v\end{array}\right]$, with $v \neq 0$, is rational equivalent to a vector of the form $\left[\begin{array}{l}x \\ 1\end{array}\right]$.

Definition 3. Let's define the equivalent class:

$$
C_{x}=\left\{\boldsymbol{y} \in R^{2} \mid \mathbf{y} \sim\left[\begin{array}{c}
x \\
1
\end{array}\right], x \in R\right\}
$$

and

$$
C=\left\{\left[\begin{array}{c}
x \\
1
\end{array}\right] \mid x \in R\right\}
$$

Thus, $\boldsymbol{y} \in C_{x}$ if and only if $\boldsymbol{y}$ is a representation of $x$. We sometimes write $\left[\begin{array}{c}x \\ 1\end{array}\right]$ to represent $C_{x}$.

Definition 4. A $2 \times 2$ matrix $\left[\begin{array}{ll}a & b \\ c & d\end{array}\right]$ is a rational-form transformation if $c \neq 0$.

Definition 5. Two $2 \times 2$ matrices $A$ and $B$ are rational equivalent, written $A \sim B$, if $A=k B$ for some number $k \neq 0$.

(Note that the symbol $\sim$ used here, has a different meaning to that used in elementary row operation.)

Property 5. Two $2 \times 2$ matrices $A$ and $B$ are rational equivalent if and only if $A \boldsymbol{x} \sim B \boldsymbol{x}$ for all $\boldsymbol{x} \in C$.

Property 6. The rational equivalent for matrices is an equivalent relation

Property 7. Each rational-form transformation is rational equivalent to a matrix of the form $\left[\begin{array}{ll}a & b \\ 1 & d\end{array}\right]$.

\section{Property 8.}

(1) The rational function $f(x)=\frac{a x+b}{x+d}$ is represented by $\left[\begin{array}{ll}a & b \\ 1 & d\end{array}\right]\left[\begin{array}{l}x \\ 1\end{array}\right]$

(2) The $n^{\text {th }}$ ordered composite function of $f$ is represented by $\left[\begin{array}{ll}a & b \\ 1 & d\end{array}\right]^{n}\left[\begin{array}{l}x \\ 1\end{array}\right]$. 
We now are ready to derive the formula for the root.

Consider the equation (2) when $g(x)=x$ :

$$
f^{[n]}(x)=x .
$$

Its representation is:

$$
\left[\begin{array}{ll}
a & b \\
1 & d
\end{array}\right]^{n}\left[\begin{array}{l}
x \\
1
\end{array}\right]=k\left[\begin{array}{l}
x \\
1
\end{array}\right], \text { for some non-zero number } k
$$

We are looking for $a, b$ and $d$ so that the equation (3) is satisfied for some non-zero number $k$, and for all $x \in R$. That is, we seek to find $a, b$ and $d$ such that $\left[\begin{array}{ll}a & b \\ 1 & d\end{array}\right]^{n}=k\left[\begin{array}{ll}1 & 0 \\ 0 & 1\end{array}\right]$ for some number $k$.

Let $A=\left[\begin{array}{ll}a & b \\ 1 & d\end{array}\right]$. The characteristic values of $A$ are:

$$
\begin{aligned}
& \lambda_{1}=\frac{1}{2}\left(a+d-\sqrt{(a+d)^{2}-4(a d-b)}\right) \\
& \lambda_{2}=\frac{1}{2}\left(a+d+\sqrt{(a+d)^{2}-4(a d-b)}\right)
\end{aligned}
$$

Suppose that $\lambda_{1} \neq \lambda_{2}$, then the matrix $A$ is diagonalizable. There is an invertible matrix $P$ such that:

$$
A=P D P^{-1}
$$

where $D=\left[\begin{array}{cc}\lambda_{1} & 0 \\ 0 & \lambda_{2}\end{array}\right]$. Then we have

$$
A^{n}=P D^{n} P^{-1}
$$

Since $D^{n}=\left[\begin{array}{cc}\lambda_{1}^{n} & 0 \\ 0 & \lambda_{2}^{n}\end{array}\right]$, demanding that $A^{n}$ be a scalar matrix, it implies that $\lambda_{1}^{n}=\lambda_{2}^{n}$. Therefore, the problem now becomes finding $a, b$ and $d$ so that $\lambda_{1}^{n}=\lambda_{2}^{n}$.

If $\lambda_{1}=\lambda_{2}$, the matrix $A$ is not diagonalizable, therefore there is no solution for the case $n=1$.

For $n=2, \quad \lambda_{1}^{2}=\lambda_{2}^{2}$ and $\lambda_{1} \neq \lambda_{2}$ if and only if $a+d=0$. Let $d=-a$, then

$$
\lambda_{1}^{2}=\lambda_{2}^{2}=-(a d-b)=-\left(-a^{2}-b\right)=a^{2}+b .
$$

Therefore $A=\left[\begin{array}{cc}a & b \\ 1 & -a\end{array}\right]$. 
The functional second root of the identity function in the rational form is

$$
f(x)=\frac{a x+b}{x-a}
$$

for any real number $a$ and $b$ such that $b \neq-a^{2}$. This function is also a functional $n^{\text {th }}$ root of the identity function when $n$ is even.

We will investigate for the general case $n>1$.

We have that $\lambda_{1} \neq \lambda_{2}$ if and only if $(a+d)^{2}-4(a d-b) \neq 0$. If $(a+d)^{2}-$ $4(a d-b)>0$ then $\lambda_{1}$ and $\lambda_{2}$ are real, $\lambda_{1}^{n}=\lambda_{2}^{n}$ and $\lambda_{1} \neq \lambda_{2}$ if and only if $a+d=0$ and $n$ is even. This case leads to the done case $n=2$, which gives the solution (5).

Now, suppose that $(a+d)^{2}-4(a d-b)<0$, then $\lambda_{1}$ and $\lambda_{2}$ are complex numbers. We write

$$
\begin{aligned}
& \lambda_{1}=\frac{1}{2}\left(a+d-i \sqrt{4(a d-b)-(a+d)^{2}}\right) \\
& \lambda_{2}=\frac{1}{2}\left(a+d+i \sqrt{4(a d-b)-(a+d)^{2}}\right)
\end{aligned}
$$

Let $\lambda_{1}^{n}=\lambda_{2}^{n}=M$, for some real number $M$. We write in the complex form:

$$
M=|M|(\cos (2 k \pi+\theta)+i \sin (2 k \pi+\theta))
$$

where $|M|=\left|\lambda_{1}^{n}\right|=\left|\lambda_{1}\right|^{n}, k=0,1,2, \ldots$, and $\theta=0$ or $\pi$ according to whether $M$ is positive or negative.

From (6a) and (6b), note that $a d-b>0$, we have

$$
\left|\lambda_{1}\right|=\left|\lambda_{2}\right|=\frac{1}{2} \sqrt{(a+d)^{2}+4(a d-b)-(a+d)^{2}}=\sqrt{a d-b}
$$

Then

$$
|M|=(a d-b)^{n / 2}
$$

From (7), the de'Moivre theorem gives $\lambda_{1}$ and $\lambda_{2}$ in the form:

$$
\lambda=\sqrt{a d-b}\left(\cos \left(\frac{2 k \pi+\pi}{n}\right)+i \sin \left(\frac{2 k \pi+\pi}{n}\right)\right)
$$

or

$$
\lambda=\sqrt{a d-b}\left(\cos \left(\frac{2 k \pi}{n}\right)+i \sin \left(\frac{2 k \pi}{n}\right)\right)
$$

for some number $k=0,1,2, \ldots, n-1$. 
Compare (6a) and (6b) to (10), then we have:

$$
\frac{1}{2}(a+d)=\sqrt{a d-b} \cos \left(\frac{2 k \pi+\pi}{n}\right) \text { or } \frac{1}{2}(a+d)=\sqrt{a d-b} \cos \left(\frac{2 k \pi}{n}\right)
$$

which can be combined to be single equation:

$$
\frac{1}{2}(a+d)=\sqrt{a d-b} \cos \left(\frac{k \pi}{n}\right), k=0,1,2, \ldots, 2 n-1
$$

If $\cos \left(\frac{k \pi}{n}\right)=0$, we have $a+d=0$. Then we have (5).

If $\cos \left(\frac{k \pi}{n}\right)= \pm 1$, then the imaginary part is zero, which is not the case. In other cases, we have

$$
b=a d-\frac{(a+d)^{2}}{4 \cos ^{2}\left(\frac{k \pi}{n}\right)}
$$

for some integer $k=1,2, \ldots, \leq \frac{n}{2}$ (Since for $k>\frac{n}{2}$ the value of $\cos ^{2}\left(\frac{k \pi}{n}\right)$ is repeated). The number $k$ should be relatively prime to $n$, since otherwise the function $f$ will not be the minimal $n^{\text {th }}$ root.

From the above analysis, the conclusion will be in accordance with the following theorem:

Theorem 1. The rational functional $n^{\text {th }}$ root of the identity function is $f(x)=\frac{a x+b}{x+d}$, where $a, b$ and $d$ satisfy the equation (11).

The result from our analysis may look a little different from the solution given by the formula (d1), but with a little work, one can show that they actually coincide.

The following is a list of the functional $n^{\text {th }}$ root of unity for $n \leq 10$. Note that for $n>2, \quad a+d \neq 0$; for $n=7$ and 9 , the exact value of $\cos \left(\frac{k}{n}\right)$ is not known.

\section{Functional Roots of the Form $\frac{a x^{2}+b x+c}{x^{2}+d x+e}$}

Now we look for the rational solution of the form $f(x)=\frac{a x^{2}+b x+c}{x^{2}+d x+e}$. For example the function $f(x)=\frac{2 x^{2}-13 x+21}{x^{2}-2 x-3}$ is the functional cube root of $x$. 
Table 1: List of the functional $n^{\text {th }}$ root of unity

\begin{tabular}{c|c|c}
\hline$n$ & $f(x)$ & $a=1, d=1$ \\
\hline 2 & $\frac{a x+b}{x-a}, b \neq-a^{2}$ & $\frac{x+b}{x-1}, b \neq-1$ \\
\hline 3 & $\frac{a x+a d-(a+d)^{2}}{x+d}$ & $\frac{x-3}{x+1}$ \\
\hline 4 & $\frac{a x+a d-\frac{1}{2}(a+d)^{2}}{x+d}$ & $\frac{x-1}{x+1}$ \\
\hline 5 & $\frac{a x+a d-\frac{1}{2}(3 \pm \sqrt{5})(a+d)^{2}}{x+d}$ & $\frac{x-(5 \pm 2 \sqrt{5})}{x+1}$ \\
\hline 6 & $\frac{a x+a d-\frac{1}{3}(a+d)^{2}}{x+d}$ & $\frac{x-\frac{1}{3}}{x+1}$ \\
\hline 8 & $\frac{a x+a d-\frac{1}{2}(2 \pm \sqrt{2})(a+d)^{2}}{x+d}$ & $\frac{x-(3 \pm 2 \sqrt{2})}{x+1}$ \\
\hline 10 & $\frac{a x+a d-\frac{1}{10}(5 \pm \sqrt{5})(a+d)^{2}}{x+d}$ & $\frac{x-\frac{1}{5}(5 \pm 2 \sqrt{5})}{x+1}$ \\
\hline
\end{tabular}

This fact can be easily seen by reducing the fraction to be $\frac{2 x-7}{x+1}$ which is in the linear rational form, where $a=2$ and $d=1$. We want to investigate this function to see whether the functional roots are of this type. Note that analyzing this function cannot be done by using the matrix representation, since the $3 \times 3$ matrix does not match the transformation.

Before analyzing the function, we should mention here the important property of all the functional roots of unity.

Firstly, we investigate the set $\mathrm{S}$ in which the function satisfies the equation for all $x \in S$. Let $D$ be the domain and $R$ the range of a root. By definition of a composite function, we have $R \subseteq D$. Suppose that $x \in D-R$, then there is no $x \in R$, such that $x=f\left(f^{[n-1]}(x)\right)=f(x)$. Therefore the set $\mathrm{S}$ must be defined so that it is both the domain and the range of $f$.

Property 3.1. The domain and range of a functional root are the same.

Property 3.2. Each root is invertible for all $x$ in its domain.

This fact can be seen easily from $f\left(f^{[n-1]}(x)\right)=x=f^{[n-1]}(f(x))$ which implies $f^{-1}(x)=f^{[n-1]}(x)$. Therefore, any functional root must be a one-toone function.

Now we are ready to analyze the function $f(x)=\frac{a x^{2}+b x+c}{x^{2}+d x+e}$, where the fraction cannot be reduced into a linear form. Let $S$ be the set of extended 
real numbers; that is the set of real numbers with $\infty$ and $-\infty$. The value of function at $\infty$ or $-\infty$ can be defined according to the limit at $\infty$ or $-\infty$. Also $f\left(a^{-}\right)$can be defined to be $\infty$ or $-\infty$ according to the left limit, and $f\left(a^{+}\right)$the right limit of $f$ at $a$.

Case 1: $a \neq 0$, and the numerator $a x^{2}+b x+c$ can be factored into real linear function. Then there are two real zeros, which implies that the function is not one-to-one. Thus, $f(x)$ is not a functional root. If the factors are the same, then the sign of the rational function near that zero are the same. There must be two points near the zero such that the rational function assumes the same value. Thus, the function is not one-to-one, and therefore not a functional root.

Case 2: $a \neq 0$, and the numerator $a x^{2}+b x+c$ cannot be real factored. Then the numerator must be positive for all real numbers, or negative for all real numbers.

Case 2.1 : If the denominator can be real factored, then there are two points $s$ and $t$, such that the one-side limit at those points is $\infty$ or $-\infty$. Since there are four one-side limits with only two values $\infty$ or $-\infty$, then there are two limits which approach the same value $\infty$ or $-\infty$. Since the function $f$ is continuous everywhere, with the exception of those two points, then there exist two distinct points, one near $s$, and the other near $t$, where $f$ assumes the same value. Thus, the function $f$ is not one-to-one.

If $s=t$, then the conclusion will be the same, since there are two one-side limits that approach only one value $\infty$.

Case 2.2 : If the denominator cannot be real factored, then the function $f(x)$ must be either positive or negative for all real numbers. Then the range of the function is not the set of extended real numbers. Thus, $f$ is not the root of unity.

Case 3: $a=0$, so the function $f$ is $f(x)=\frac{b x+c}{x^{2}+d x+e}$. If the denominator can be real factored, then by the reason as stipulated in $2.1, f$ is not one-to-one. If the denominator cannot be real factored, then the function is bounded since there is no discontinuity point, and $\lim _{x \rightarrow-\infty} f(x)=\lim _{x \rightarrow \infty} f(x)=0$. Therefore the range of the function is not the set of extended real numbers.

Case 4 : Consider the function of the form $\frac{a x^{2}+b x+c}{x+e}$ where $a \neq 0$. Observe that $\lim _{x \rightarrow-\infty} f(x)$ and $\lim _{x \rightarrow \infty} f(x)$ approach the different values $\infty$ or $-\infty$, and also $\lim _{x \rightarrow-e^{-}} f(x)$ and $\lim _{x \rightarrow-e^{+}} f(x)$ approach the different values $\infty$ or $-\infty$. Therefore, in accordance to reasons similar to Case 2.1, the function is not one-to-one. 
From our analysis, a conclusion can be drawn about the fractions of quadratic functions, and the fractions of polynomials degree greater than one, except where they can be reduced to the fractions of linear functions.

Theorem 2. There is no functional root of unity in the rational form of quadratic functions.

Corollary. There is no functional root of unity in the rational form of polynomials degree greater than one.

\section{Conclusions}

There are infinite solutions for Babbage's equation. Although the rational form solution of Babbage's equation is well known, its derivation given by Boole [1] is somewhat complicated. In this report, we showed the derivation of the rational solutions by mean of special linear transformation. The derived formula is equivalent to the old formula, but it is a little different in its form. Examples of particular solutions for some $\mathrm{n}$ were also provided.

Analyzing the functions of the rational form of quadratics shows that there is no solution in that form, except that can be reduced into fractions of linear functions. These results can also be extended to the fractions of polynomials of degree greater than one.

\section{Acknowledgments}

This work was completed with the support of the Faculty of Science Research Fund, Burapha University.

\section{References}

[1] G. Boole, A Treatise on the Calculus of Finite Differences, Cambridge, (Printed by C. J. Clay, M.A. at the University Press) (1860), 208-216.

[2] B. Choczewski (Gliwice), On continuous solutions of some functional equations of the n-th Order, Annales Polonici Mathematici XI (1961), 123 - 132.

[3] G. M. Ewing and W. R. Utz, Continuous Solutions of the Functional Equation $f^{n}(x)=f(x)$, Canada. J. Math. 5 (1953), 101-103. 
A. Dhamacharoen, P. Kasempin

[4] R. Isaacs, Iterates of Fractional Order, Canad. J. Math. 2 (1950), 409-416.

[5] M. Kuczma (Krakow), On Some Funtional Equation Containing Iterations of the Unknown Function, Annales Polonici Mathematici XI (1961), 1 - 5.

[6] M. Kuczma (Krakow), On the Funtional Equation $\phi^{n}(x)=g(x)$, Annales Polonici Mathematici XI (1961), 161 - 175.

[7] A. Mach, On some Functional Equations Involving Babbage Equation, Result in Mathematics 51(1-2) (2007), 97-106.

[8] A. D. Polyanin and A. V. Manzhirov, Handbook of Integral Equations: Exact Solutions (Supplement some functional equations), Faktorial, Moscow, (1998).

[9] J. F. Ritt, On Certain Real Solutions of Babbage's Functional Equation, Annals of Mathematics, Second Series,17(3) (1916), 113-122.(Stable URL: http://www.jstor.org/stable/2007270).

[10] C. G. Small, Functional Equations and How to Solve Them, Springer Science Business Media, LLC, (2007).

[11] F. J. McCarthy,Functional nth Roots of Unity, The Mathematical Gazette, 64(428), (1980). 